\title{
Rumen microbial responses to supplemental nitrate. I. Yeast growth and protozoal chemotaxis in vitro as affected by nitrate and nitrite concentrations
}

\author{
Y. Roman-Garcia, ${ }^{1}$ B. A. Wenner, ${ }^{1}$ C. M. Welty, ${ }^{1 *}$ B. K. Wagner, ${ }^{1}$ J. E. Plank, ${ }^{1} \dagger$ R. A. Meller, ${ }^{1}$ S. J. Waits, ${ }^{1}$ \\ A. M. Gehman, ${ }^{2}$ and J. L. Firkins ${ }^{1} \ddagger$ \\ ${ }^{1}$ Department of Animal Sciences, The Ohio State University, 2029 Fyffe Ct., Columbus 43210 \\ ${ }^{2}$ Alltech, 3031 Catnip Hill Pike, Nicholasville, KY 40356
}

\section{ABSTRACT}

Nitrates have been fed to ruminants, including dairy cows, as an electron sink to mitigate $\mathrm{CH}_{4}$ emissions. In the $\mathrm{NO}_{3}{ }^{-}$reduction process, $\mathrm{NO}_{2}{ }^{-}$can accumulate, which could directly inhibit methanogens and some bacteria. However, little information is available on eukaryotic microbes in the rumen. Protozoa were hypothesized to enhance nitrate reductase but also have more circling swimming behavior, and the yeast Saccharomyces cerevisiae was hypothesized to lessen $\mathrm{NO}_{2}{ }^{-}$accumulation. In the first experiment, a culture of $S$. cerevisiae strain 1026 was evaluated under 3 growth phases: aerobic, anoxic, or transition to anoxic culture. Each phase was evaluated with a control or 1 of 3 isonitrogenous doses, including $\mathrm{NO}_{3}^{-}, \mathrm{NO}_{2}^{-}$, or $\mathrm{NH}_{4}{ }^{+}$replacing peptone in the medium. Gas head phase, $\mathrm{NO}_{3}{ }^{-}$, or $\mathrm{NH}_{4}{ }^{+}$did not influence culture growth, but increasing $\mathrm{NO}_{2}{ }^{-}$concentration increasingly inhibited yeast growth. In experiment 2 , rumen fluid was harvested and incubated for $3 \mathrm{~h}$ in 2 concentrations of $\mathrm{NO}_{3}{ }^{-}, \mathrm{NO}_{2}{ }^{-}$, or sodium nitroprusside before assessing chemotaxis of protozoa toward glucose or peptides. Increasing $\mathrm{NO}_{2}{ }^{-}$concentration decreased chemotaxis by isotrichids toward glucose or peptides and decreased chemotaxis by entodiniomorphids but only toward peptides. Live yeast culture was inhibited dose-responsively by $\mathrm{NO}_{2}{ }^{-}$and does not seem to be a viable mechanism to prevent $\mathrm{NO}_{2}{ }^{-}$accumulation in the rumen, whereas a role for protozoal nitrate reductase and $\mathrm{NO}_{2}^{-}$influencing signal transduction requires further research.

Received June 25, 2018.

Accepted November 27, 2018.

*Current location: Renaissance Nutrition, PO Box 229, Roaring Spring, PA 16673.

†Current location: Department of Statistics, The Ohio State University, Columbus 43210.

$\ddagger$ Corresponding author: firkins.1@osu.edu
Key words: nitrate, yeast culture, protozoal chemotaxis, methane emission

\section{INTRODUCTION}

Nitrates have been fed to mitigate enteric $\mathrm{CH}_{4}$ emission from ruminants. Some rumen microbes can reduce dietary $\mathrm{NO}_{3}{ }^{-}$to $\mathrm{NO}_{2}{ }^{-}$to $\mathrm{NH}_{3}$, using nitrate and nitrite reductases, which can harness reducing power from aqueous $\mathrm{H}_{2}$ and thermodynamically outcompete methanogenesis (Hristov et al., 2013a; Latham et al., 2016). Before feeding nitrates, the rumen environment must have time to adapt, primarily to decrease $\mathrm{NO}_{2}{ }^{-}$ accumulation enough to cause methemoglobinemia (Hristov et al., 2013b; Lund et al., 2014; Latham et al., 2016). In addition, $\mathrm{NO}_{3}{ }^{-}$reduction intermediates, $\mathrm{NO}_{2}{ }^{-}$and $\mathrm{NO}$, might be toxic to methanogens (Latham et al., 2016; Yang et al., 2016). With proper adaptation and near complete $\mathrm{NO}_{2}{ }^{-}$reduction to $\mathrm{NH}_{3}$ for bacterial assimilation, $\mathrm{NO}_{3}{ }^{-}$could replace urea in the diet (Latham et al., 2016).

Castello et al. (2006) report that yeast mitochondria can reduce $\mathrm{NO}_{2}{ }^{-}$to $\mathrm{NO}$ via cytochrome c oxidase, particularly when transitioning from aerobic to anoxic conditions, which occurs in the rumen provided that electron donors are available. In fact, respiration of $\mathrm{O}_{2}$ is a likely mode of action for live yeast to stimulate growth of anaerobic bacteria such as cellulolytics (Newbold et al., 1996; Jeyanathan et al., 2014). If Saccharomyces cerevisiae reduces $\mathrm{NO}_{2}{ }^{-}$, then risk of methemoglobinemia might be lessened when $\mathrm{NO}_{3}{ }^{-}$salts are fed. Yeast have mixed results when studied for a role in methanogenesis (Jeyanathan et al., 2014), perhaps because of a combination of these varying responses on methanogens and $\mathrm{H}_{2}$-producing bacteria.

In contrast with yeast, the ruminal ciliate protozoa have been more routinely associated with enhanced methanogenesis because acetate or butyrate fermentation occurs via hydrogenosomes or mitosomes (Park et al., 2017) to produce $\mathrm{H}_{2}$ or formate, thus providing 
precursors for intra- and extracellular methanogenic archaea (Yang et al., 2016). Protozoa appear to contribute significant nitrate reductase activity (Latham et al., 2016; Yang et al., 2016). Because sodium nitroprusside (Snp) generates NO to activate protein kinase $\mathrm{G}$ and directional motility in ruminal ciliates (Diaz et al., 2014a), $\mathrm{NO}_{2}{ }^{-}$was hypothesized to activate $\mathrm{NO}$ signal transduction, which to our knowledge has not been addressed for ciliates. Moreover, $\mathrm{NO}_{2}^{-}$could inhibit protozoa directly (Asanuma et al., 2015), which could decrease abundance or, alternatively, maintain abundance but increase stress responses that actually could increase their need for substrate as acquired through chemotaxis.

Our objectives were to address dose-responsive effects of $\mathrm{NO}_{3}{ }^{-}$and $\mathrm{NO}_{2}{ }^{-}$on yeast growth rate and on motility behavior of isotrichid and entodiniomorphid ciliate protozoa. Potential responses of live yeast culture to mitigate $\mathrm{NO}_{2}{ }^{-}$accumulation and on protozoal abundance in continuous cultures were assessed in the companion paper (Welty et al., 2019). In experiment 1, we hypothesized that yeast growth would not be affected by $\mathrm{NO}_{3}{ }^{-}$or $\mathrm{NH}_{3}$, but $\mathrm{NO}_{2}{ }^{-}$would either inhibit growth by disrupting electron transport or stimulate growth if $\mathrm{NO}_{2}{ }^{-}$acts as a substitute for $\mathrm{O}_{2}$ as a terminal electron acceptor when transitioning to anoxic conditions. In experiment 2, we hypothesized that $\mathrm{NO}_{3}{ }^{-}$would have no effect, but $\mathrm{NO}_{2}^{-}$would inhibit protozoa motility and attenuate chemotaxis as mediated by NO signaling.

\section{MATERIALS AND METHODS}

\section{Experiment 1}

Live Yeast Culture Conditions. Saccharomyces cerevisiae 1026 was obtained from the National Collection of Yeast Cultures (Norwich, UK). Cultures were grown in a $39^{\circ} \mathrm{C}$, shaking incubator in liquid yeast peptone dextrose medium, both aerobically and anaerobically (Green and Mochle, 1999), in 3 replicate incubations. In a $3 \times 3 \times 3$ factorial arrangement of treatments 25,50 , and $75 \%$ of total $\mathrm{N}$ was replaced isonitrogenously by $\mathrm{NaNO}_{3}, \mathrm{NaNO}_{2}$, or $\left(\mathrm{NH}_{4}\right)_{2} \mathrm{SO}_{4}(3$ concentrations of 3 different treatments) by removing peptone from the medium; these treatments were factorialized with 3 headspace gas phases (aerobic, transition from aerobic to anoxic, and anoxic). A common control was the 10th treatment. Hereafter, we refer to anoxic conditions in which $\mathrm{O}_{2}$ is limited (as in anaerobic conditions) but $\mathrm{NO}_{3}{ }^{-}$or $\mathrm{NO}_{2}{ }^{-}$are added. For the aerobic phase, $12.0 \mathrm{~mL}$ of each aerobic autoclaved medium was placed into 5 tubes and inoculated with $1.2 \mathrm{~mL}$ of aerobically grown S. cerevisiae 1026 within the log phase at an optical density (OD) of 0.20 (Spectronic 20D+, Waltham, MA). Aerobiosis was achieved by capping with foil to prevent contamination while allowing atmospheric $\mathrm{O}_{2}$ equilibration with the medium. For transition and anoxic phases, 10 tubes were filled with each autoclaved anaerobic medium and inoculated with yeast culture at $0.20 \mathrm{OD}$; for transition phase, 5 tubes were inoculated with aerobically grown yeast culture, but for the anoxic phase, the other 5 were inoculated with anaerobically grown yeast culture. Both transition and anoxic phases were gassed with $\mathrm{CO}_{2}$ and capped with a 1-way valve inserted into a stopper after autoclaving. Samples were incubated at $39^{\circ} \mathrm{C}$ in a shaking water bath until sampled at $0,3,6,12$, and 24 h. At respective times (staggered with inoculation), 1 tube from each treatment was removed, and $5 \mathrm{~mL}$ was placed in a glass test tube for immediate measurement of OD at $600 \mathrm{~nm}$ after which it was microscopically screened for any bacterial contamination. In the few samples in which bacterial contamination was detected, samples were excluded from analysis, although the OD appeared unaffected. Three replicate tubes were averaged per time per run. The $0-\mathrm{h}$ OD was subtracted from the $\mathrm{OD}$ of each subsequent incubation time to covariate-adjust data to a common initial OD.

Statistical Analysis. The model included treatment (nitrogen source), concentration (percentage of total median N substitution), gas phase (aerobic, transition, or anoxic), hour, and all possible interactions. Yeast growth of the mean of replicates per time was fitted by NLIN (version 8.2, SAS Institute Inc., Cary, $\mathrm{NC}): \mathrm{OD}=\mathrm{a}+\mathrm{b}\left(1-\mathrm{e}^{-\mathrm{kt}}\right)$, where $\mathrm{a}$ is the initial OD, $\mathrm{b}$ is the change from initial OD to asymptote, $\mathrm{k}$ is the first-order fractional growth rate, and $t$ is time in hours. All models converged except for some $\mathrm{NO}_{2}{ }^{-}$treatments from which growth was barely evident. These fitted values were plotted against actual OD (Figure 1). Because of the high correlation between $\mathrm{b}$ and $\mathrm{k}$ terms, actual means for OD were analyzed by ANOVA as a $3 \times 3$ $\times 3$ factorial arrangement of treatments (3 gas phases and the 3 isonitrogenous treatments $\left(\mathrm{NO}_{3}{ }^{-}, \mathrm{NO}_{2}{ }^{-}\right.$, and $\mathrm{NH}_{4}{ }^{+}$) at each of their 3 substitution percentages of peptone), including all possible interactions, with run (3 replicate incubations) as a fixed effect block. Significance was declared at $P \leq 0.05$. Trends were declared at $0.05<P \leq 0.10$.

\section{Experiment 2}

Protozoal Chemotaxis Assay. Rumen fluid was collected from at least 3 different locations (central mat, caudal, and ventral sites) from a ruminally cannulated multiparous Jersey cow that was unadapted 

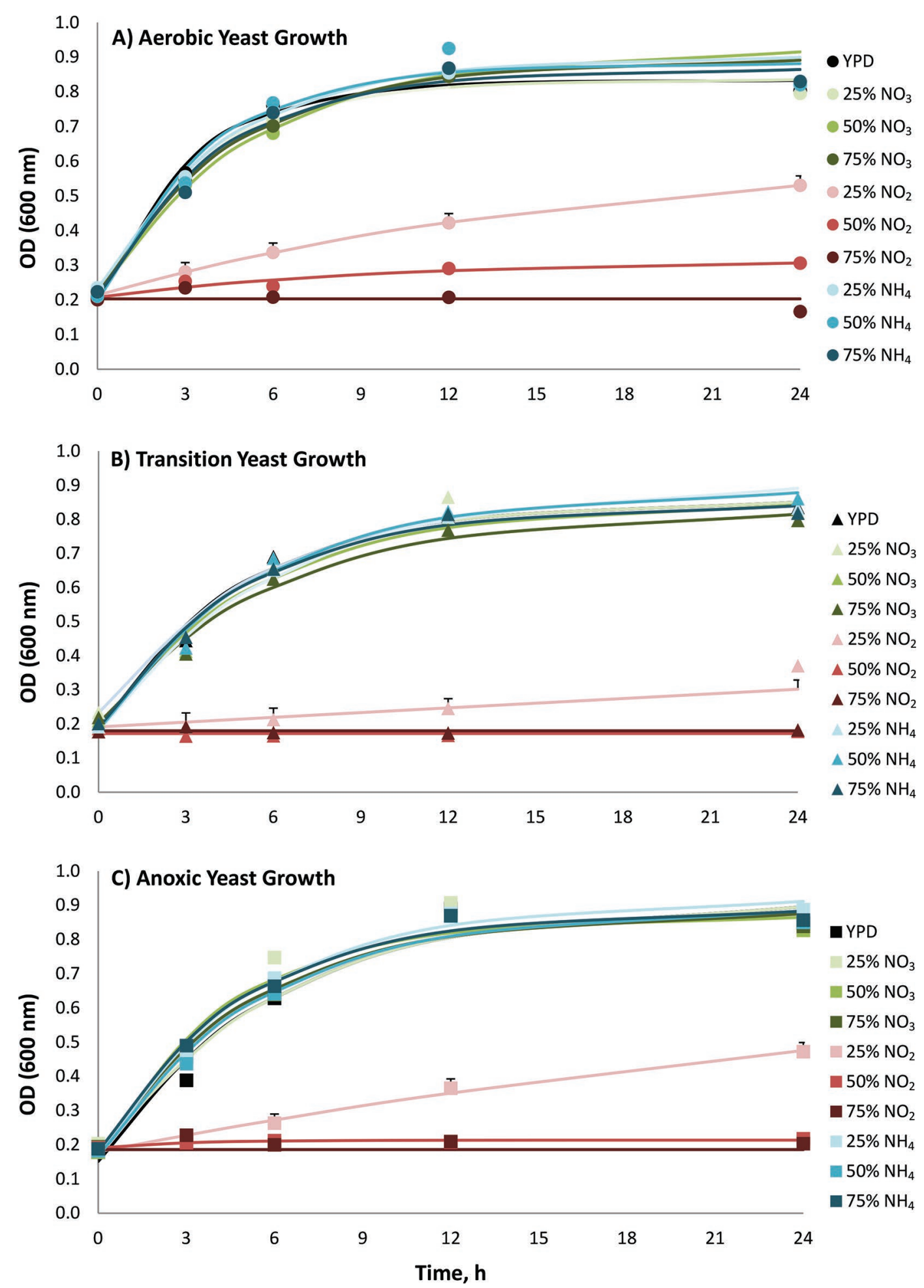

Figure 1. In experiment 1, growth of Saccharomyces cerevisiae (measured by optical density, OD) in media that were aerobic (A), transitioning from aerobic to anoxic $(\mathrm{B})$, or anoxic $(\mathrm{C})$ with $0,25,50$, or $75 \%$ of media $\mathrm{N}$ isonitrogenously replaced by nitrate $\left(\mathrm{NO}_{3}\right)$, nitrite $\left(\mathrm{NO}_{2}\right)$, or ammonium $\left(\mathrm{NH}_{4}\right)$ in comparison to yeast peptone dextrose (YPD) control. Nitrogen source, percentage, gas phase, hour, and all interactions were $P<0.01$ (data not shown) for all OD responses. When the control was excluded, there was no gas phase $\times \mathrm{N}$ source $\times \mathrm{N}$ percentage $\times$ hour interaction $\left(P>0.15\right.$; data not shown). When analyzed by $\mathrm{N}$ source, the $\mathrm{NO}_{2}{ }^{-}$treatment had a $\mathrm{N}$ percentage $\times$ hour interaction $(P<$ $0.01)$, but this interaction was $P>0.15$ for other $\mathrm{N}$ sources. 
to dietary $\mathrm{NO}_{3}{ }^{-}$. The cow was housed at the Waterman Dairy (The Ohio State University, Columbus) according to an approved animal usage protocol. After straining through 2 layers of cheesecloth, ruminal fluid was diluted 1 to 3 with simplex buffer (made without cysteine; Dehority, 1998). After gassing with $\mathrm{O}_{2}$-free $\mathrm{CO}_{2}$ and incubation for $30 \mathrm{~min}$ in a separatory funnel in a $39^{\circ} \mathrm{C}$ incubator oven, foamy particulate matter was aspirated off the top of the fluid. Then, a sample was taken to establish a baseline protozoal concentration $(0 \mathrm{~h})$, and $25 \mathrm{~mL}$ was inoculated into a series of $50-\mathrm{mL}$ tubes with 1-way valves while under $\mathrm{CO}_{2}$. Individual tubes were pre-incubated for $3 \mathrm{~h}$ with one of the following chemosensory compounds (final concentrations): $0.9 \%$ saline (control), $500 \mu M \mathrm{Snp}, 5.68 \mathrm{mM} \mathrm{NaNO}{ }_{3}$, $17.03 \mathrm{~m} M \mathrm{NaNO}_{3}, 5.68 \mathrm{~m} M \mathrm{NaNO}_{2}$, or $17.03 \mathrm{~m} M$ $\mathrm{NaNO}_{2}$. The Snp concentration in $0.1 \%$ dimethyl sulfoxide was based on Diaz et al. (2014c). The $5.68 \mathrm{mM}$ $\mathrm{NO}_{3}{ }^{-}$and $\mathrm{NO}_{2}{ }^{-}$dosages are related to the concentration in the companion paper (Welty et al., 2019) that we expected when daily feeding doses were dissolved into the average fermentor working volume. In the current chemotaxis study, dosing of each treatment into individual tubes was staggered in 5-min intervals and vessels were shaken in a water bath at $39^{\circ} \mathrm{C}$ to ensure that all treatments had the same average time after ruminal fluid collection.

After the 3 -h pre-incubation in a water bath at $39^{\circ} \mathrm{C}, 40 \mu \mathrm{L}$ of sample from each incubation tube was wet-mounted, and 9 videos of $10 \mathrm{~s}$ each were taken for assessment of motility to be described subsequently. After subsampling for videography and still under a $\mathrm{CO}_{2}$ stream, a 20-mL aliquot of each incubated tube was transferred to a $50-\mathrm{mL}$ beaker and sealed with parafilm. Capillary tubes were preloaded with $0.9 \%$ saline, $1 M$ glucose dissolved in $0.9 \%$ saline, or $1 \mathrm{~g} / \mathrm{L}$ of soluble soy protein isolate dissolved in $0.9 \%$ saline; then, tubes were sealed and placed in duplicate in the beakers for $20 \mathrm{~min}$. All treatment justifications, dosages, and procedures were as described by Diaz et al. (2014c). After combining the contents of duplicate capillary tubes, isotrichid and entodiniomorphid protozoa were counted by wet mounting capillary contents on a disposable glass slide marked with a $5 \times 5$ grid. Two replications were done per day, and the experiment was repeated over $3 \mathrm{~d}$, with the treatment order arranged so that time after collection from the donor cow was equalized for all treatments. Entire ocular fields of a grid with 25 viewing areas were counted using a $15 \times$ objective and a $10 \times$ lens. The slide was rotated once, and the count was repeated.

The 10-s videos were imported into ImageJ (Schneider et al., 2012) and analyzed similarly to motility assessments in Wenner et al. (2018). Immotility was assessed by trained personnel who were blinded to treatment. All protozoa in the first frame of each video were distinguished as entodiniomorphids or isotrichids, counted, and tracked during the remaining frames of the video. Any protozoa that entered in subsequent frames were excluded. The nonmotile cells were expressed as a percentage of original (in the first frame) protozoa per video. For analysis of swimming speed of the motile protozoa, videos were converted to a digital stack in grayscale, nonmoving cells were subtracted from the stack, and moving cells were identified by the Tetratracker plugin. Exclusion parameters for these videos were limited to cells with an area between 375 and $25,000 \mu^{2}$, a velocity of $25 \mu \mathrm{m} / \mathrm{s}$ or less, and area change of less than $33 \mu^{2}$. When tracking entodiniomorphids, only cells that were in the screen for at least $1 \mathrm{~s}$ were considered; in contrast, most isotrichids were not in the screen for more than $1 \mathrm{~s}$ but all were considered because of their lower numbers. Cells with an area between 1,000 and $3,125 \mu^{2}$ were considered entodiniomorphids, and those between 3,125 to 25,000 $\mu \mathrm{m}^{2}$ were considered isotrichids. These ranges were obtained from minimum, mean, and maximum sizes reported by Dehority (1993). The incidence of genus Dasytricha or Ophryoscolex that were less than or greater than $3,125 \mu^{2}$, respectively, were nominal and randomly distributed (data not shown). Total speed (including turning) and displacement speed (excluding turning) were pooled for the 9 videos such that a mean and standard deviation were derived per tube (i.e., tube was the experimental unit).

Statistical Analysis. Counts of protozoa in the beakers and in capillary tubes were $\log _{10}$-transformed to normalize data, which were covariate-adjusted for individual beakers as justified by Diaz et al. (2014b) except that beaker counts were used instead of unsealed capillary tubes. For samples that had no isotrichids, the count was manually converted to 0.01 (deemed a trivial value) to allow log-transformation. The counts were analyzed by the MIXED procedure of SAS (SAS Institute, 1999) with a model that included the random block for replication and the fixed effects of 3-h incubation treatment (in beakers), capillary tube treatments, and their interactions. The means were compared by 5 pre-planned contrasts: control versus the mean of all $4 \mathrm{NO}_{3}{ }^{-}$and $\mathrm{NO}_{2}{ }^{-}$beaker treatments, control versus Snp, main effects of $\mathrm{NO}_{3}{ }^{-}$versus $\mathrm{NO}_{2}{ }^{-}$(both sources averaged over dose), dose concentration (5.68 or 17.03 $\mathrm{m} M$; averaged over $\mathrm{NO}_{3}^{-}$and $\mathrm{NO}_{2}^{-}$sources), and an interaction between $\mathrm{NO}_{3}{ }^{-}$versus $\mathrm{NO}_{2}{ }^{-}$and their 2 doses. Capillary tube treatments were separated by Ftest-protected least significant difference. For motility, 
the PROC MIXED procedure of SAS (SAS Institute, 1999) was used with a model that included the random block of run and included weights of 1 /standard deviation. Significance was declared at $P \leq 0.05$; and trends, $0.05<P \leq 0.10$.

\section{RESULTS AND DISCUSSION}

\section{Experiment 1}

Nitrogen source, percentage, gas phase, hour, and all interactions were $P<0.01$ (data not shown) for all OD responses for $S$. cerevisiae cultures. When the control was excluded, there was no gas phase $x \mathrm{~N}$ source $\times$ $\mathrm{N}$ percentage $\times$ hour interaction $(P>0.15$; data not shown). When analyzed by $\mathrm{N}$ source, the $\mathrm{NO}_{2}{ }^{-}$treatment had a $\mathrm{N}$ percentage $\times$ hour interaction $(P<$ 0.01 ), but this interaction was $P>0.15$ for other $\mathrm{N}$ sources. Results were inconsistent for gas phase over time, and most of the main effect of treatment was from the $\mathrm{NO}_{2}{ }^{-}$treatment being markedly less (especially with increasing $\mathrm{N}$ percentage) than the other treatments, which is clearly evident by growth plots (Figure 1). These plots also illustrate dose-dependent inhibition by $\mathrm{NO}_{2}^{-}$over the entire incubation time without any visible differences between yeast peptone dextrose medium control, $\mathrm{NO}_{3}{ }^{-}$, and $\mathrm{NH}_{4}{ }^{+}$treatments. The concentration of $\mathrm{NO}_{2}{ }^{-}$in this experiment for 25, 50 , and $75 \%$ are $0.071,0.14$, and $0.21 \mathrm{M}$, respectively. Nitrate is toxic to bacteria that produce ATP by electron-transport phosphorylation mechanisms using cytochromes, whereas inconsistent toxicity to other bacteria needs further clarification (Yang et al., 2016). Those authors suggested that fungi might be inhibited by $\mathrm{NO}_{3}{ }^{-}$or $\mathrm{NO}_{2}{ }^{-}$, but to our knowledge, little is known about $\mathrm{NO}_{2}{ }^{-}$toxicity to $S$. cerevisiae. They possess cytochromes, so inhibition of ATP synthesis likely explains our results. A high dosage $(5 \mathrm{mM})$ of $\mathrm{NO}_{2}{ }^{-}$inhibited $S$. cerevisiae, apparently by inhibiting glyceraldehyde3-phosphate dehydrogenase (Hinze and Holzer, 1986). In the presence of $\mathrm{O}_{2}$, NO radicals from NO signaling are oxidized to $\mathrm{NO}_{2}{ }^{-}$, which is effluxed from the cell to prevent nitrosative stress in S. cerevisiae (Sarver and DeRisi, 2005). Under our conditions, dose-responsive inhibition by $\mathrm{NO}_{2}{ }^{-}$does not support our hypothesis that yeast would respire $\mathrm{NO}_{2}^{-}$, which we assumed would occur particularly when transitioning from aerobic to anoxic phases (Castello et al., 2006).

\section{Experiment 2}

Based on pre-incubation with the NO generator Snp (Diaz et al., 2014c), we hypothesized that $\mathrm{NO}_{2}{ }^{-}$would over-stimulate protozoal chemotaxis, thus arresting their ability to acquire substrate. Although $\mathrm{NO}_{2}{ }^{-}$also activated NO-sensitive guanylate cyclase (which is stimulated by Snp) in rat endothelial cells (Ling et al., 2015), $\mathrm{NO}_{2}^{-}$has not been assessed in ruminal ciliates (to our knowledge). Motility was assessed by a combination of percentage of motile cells and the swimming speed and directionality of the motile cells, as illustrated in Supplemental Figure S1 (https://doi.org/10 .3168/jds.2018-15274). Greater circling behavior would be in response to a chemotactic gradient as when swimming from beakers into capillary tubes filled with either glucose or peptides or as mimicked by stimulation of NO-mediated protein kinase G activity when filled with Snp (Diaz et al., 2014a).

Motility measurements were not affected $(P>0.10)$ by any of the treatment contrasts for isotrichids (Figure 2 , panel A). The total and net speeds of isotrichids are lower than those reported by Diaz et al. (2014a). However, in that study, swimming speeds were only recorded for isotrichids that did not bump into feed particles or each other; the current measurements include those cells that bump, contort, and redirect their swimming, which lessens distance traveled and therefore swimming speed.

In contrast with isotrichids, entodiniomorphids had numerous treatment effects from the 3 -h incubations (Figure 2, panel B). The saline control had a lower $(P$ $<0.01)$ percentage of immotile cells than the average of the $\mathrm{NO}_{3}{ }^{-}$and $\mathrm{NO}_{2}{ }^{-}$treatments (contrast treatment 1 vs. average of treatments 2 to 5 ). This contrast was $P$ $>0.10$ for total and net speeds of the motile cells. The Snp treatment increased $(P<0.01)$ the percentage of immotile entodiniomorphids and decreased the total $(P$ $=0.05)$ and net displacement $(P=0.05)$ speeds of those that were motile compared with control (contrast 1 vs. 6). Diaz et al. (2014a) did not detect any inhibition from Snp; however, the greater vacuolation reported by Diaz et al. (2014c) suggests a potential toxicity with extended incubation. Unfortunately, vacuolation in this experiment was not recorded but might have been more severe than the previous study. When compared with $\mathrm{NO}_{3}{ }^{-}$, the percentage of immotile entodiniomorphids more than doubled for $\mathrm{NO}_{2}^{-}$(main effect $P<0.01$ ), particularly for the higher dosage (interaction $P<0.01$ ), which had over $99 \%$ immotile cells. For those entodiniomorphids that were motile, total speed decreased $(P$ $=0.02)$ and net displacement speed tended $(P=0.06)$ to decrease for the main effect means of $\mathrm{NO}_{2}{ }^{-}$versus the main effect means of $\mathrm{NO}_{3}{ }^{-}$. Motility measurements were from cells in 3 -h incubation treatments and are assumed to represent cells that were sensitized for chemotactic swimming toward glucose or peptide gradients in capillary tubes, as discussed subsequently. 
Incubation beaker treatment $\times$ capillary tube interactions were $P=0.37$ for isotrichid counts in the capillary tubes, so only main effect means for pre-incubation beaker treatments and capillary tube chemoattractants are presented in Figure 3 (panel A). Saline was greater
$(P<0.01)$ than the average of $\mathrm{N}$ source, and there were differences in the main effects of $\mathrm{N}$ source $\left(\mathrm{NO}_{3}{ }^{-}\right.$vs. $\left.\mathrm{NO}_{2}{ }^{-} ; P<0.01\right)$ but also their dosage (5.68 vs. 17.03 $\mathrm{m} M ; P=0.03)$; however, main effects have limited meaning in the presence of the $\mathrm{N}$ source $\times$ dose inter-

A) Isotrichids

Speed of Motile Protozoa

- Immotile, \% 血 Total, $\mu \mathrm{m} / \mathrm{s}$ 目 $\mathrm{Net}_{s} \mu \mathrm{m} / \mathrm{s}$

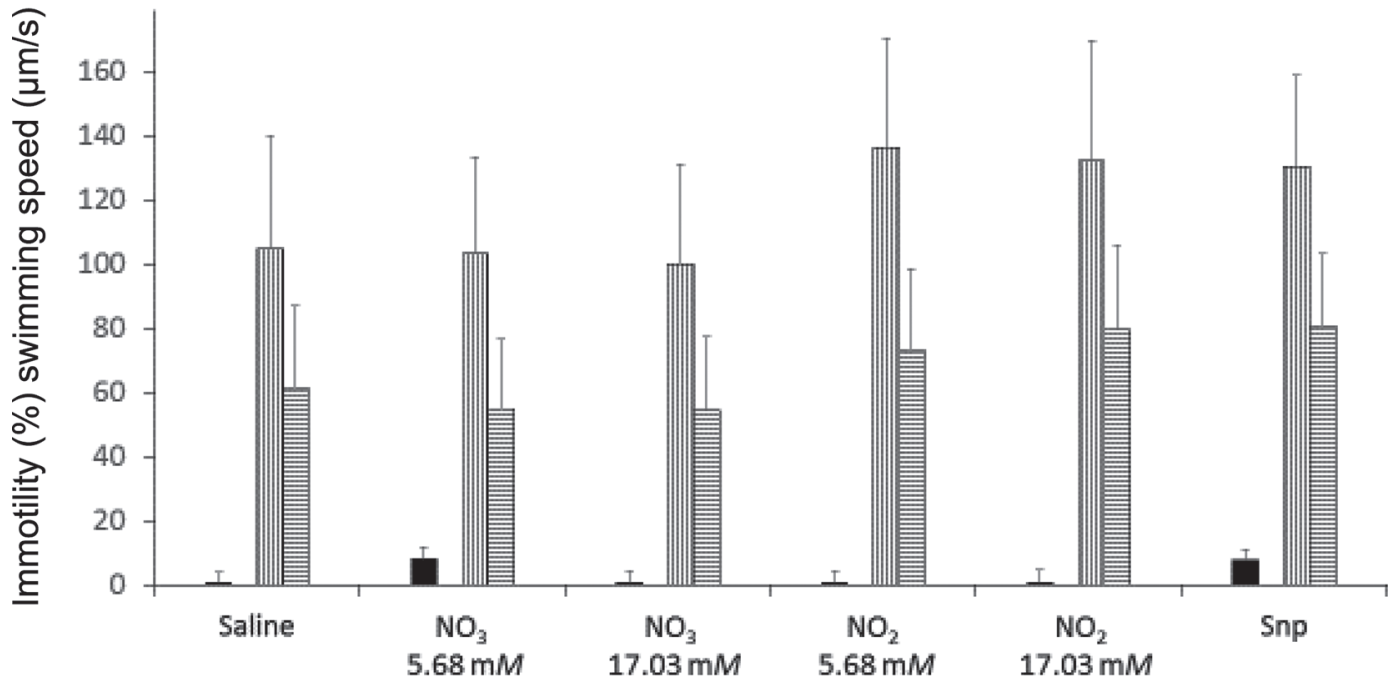

B) Entodiniomorphids

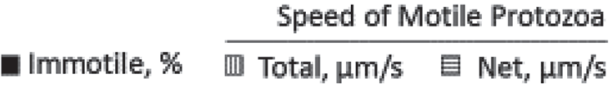

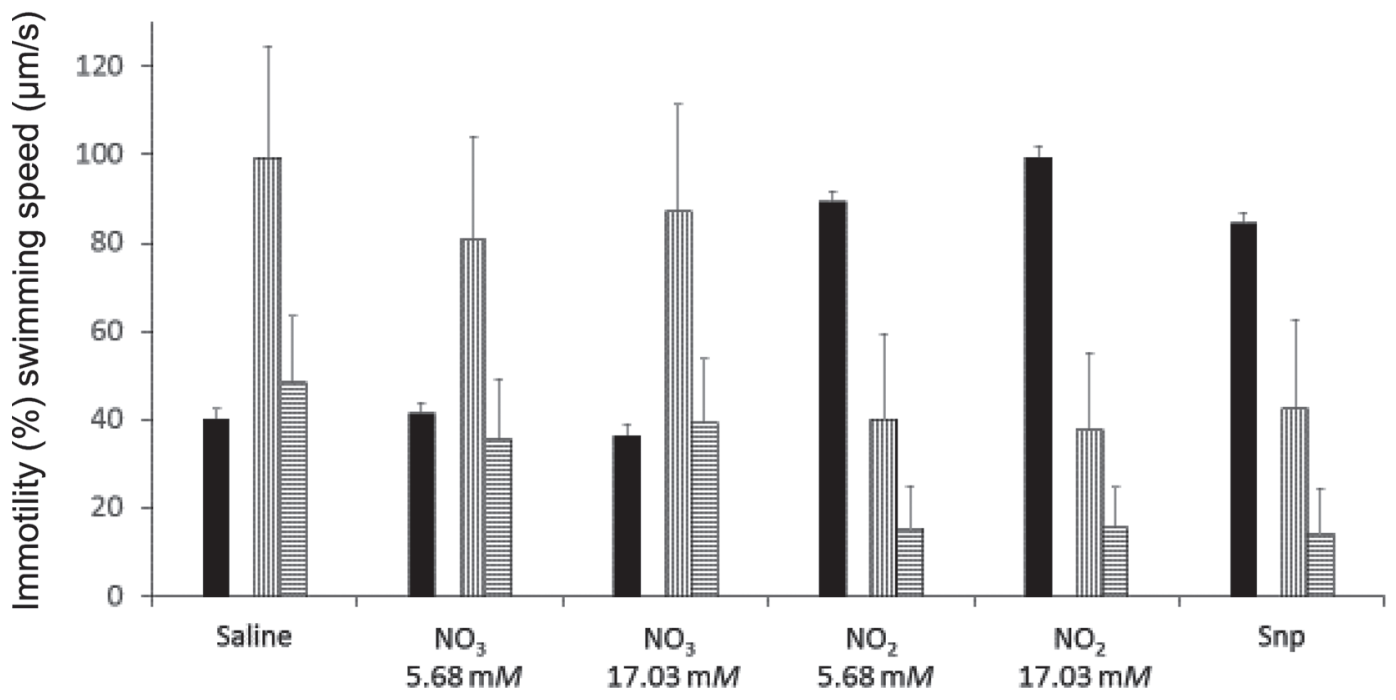

Figure 2. In experiment 2, least squares means of rumen protozoal immotility (\% of total cells that were immotile) and total (including all turns) or net (excluding turns) displacement swimming speeds of the protozoa that were motile after $3 \mathrm{~h}$ of anoxic incubation with 6 treatments: (1) saline, (2) $5.68 \mathrm{mM} \mathrm{NO}_{3}$, (3) $17.03 \mathrm{mM} \mathrm{NO}$, (4) $5.68 \mathrm{mM} \mathrm{NO}_{2}$, (5) $17.03 \mathrm{mM} \mathrm{NO}$, and (6) $500 \mu M$ sodium nitroprusside (Snp). Error bars are SEM. In panel A (isotrichids), all contrasts were $P>0.15$ except for percentage immotile for saline versus Snp $(1$ vs. $6 ; P=0.12)$. In panel B (entodiniomorphids), treatment 1 versus 2 to 5 was $P<0.01, P=0.13$, and $P>0.15$ for immotility, total speed, and net speed, respectively; saline versus Snp (1 vs. 6) was $P<0.01, P=0.05$, and $P=0.05$, respectively; the main effect of $\mathrm{NO}_{3}$ versus $\mathrm{NO}_{2}(2+3$ vs. $4+5)$ was $P<$ $0.01, P=0.02$, and $P=0.06$, respectively; the interaction of $\mathrm{NO}_{3}$ versus $\mathrm{NO}_{2}$ and dose $(2+5$ vs. $3+4)$ was $P<0.01$ for immotility; all other contrasts were $P>0.15$. 
action $(P<0.01)$. Dosage of $\mathrm{NO}_{3}{ }^{-}$had limited effect, but higher dosage of $\mathrm{NO}_{2}^{-}$decreased isotrichid counts in capillary tubes almost to zero, indicating profound blunting of chemotaxis independent of motility per se because isotrichid motility measurements were not affected (Figure 2A). In addition, Snp decreased $(P=$ 0.04) chemoattraction compared with control (Figure 3A). Diaz et al. (2014a) concluded that there was limited direct effect of Snp by isotrichids, suggesting a deemphasized need for circular swimming (compared with entodiniomorphids) for chemotactic behavior compared with their need for distance (migration) or perhaps swimming faster (as opposed to more circular) toward sugar gradients. Based on a series of eukaryotic inhibitors or activators, the model in that paper suggests that NO signaling was integrated with intracellular $\mathrm{Ca}^{2+}$ signaling. When glycogen energy stores are full, chemotaxis and motility are inhibited when isotrichids settle on the ventral wall of the reticulorumen, but when glycogen is depleted, motility is again activated for them to migrate dorsally even without a chemotactic signal. Apparently, isotrichids partition into groups coinciding with patterned meal feeding behavior by the animal, and the glycogen-depleted cells migrate in synchrony with the animal's expected meal (Diaz et al., 2014a). These migratory cells probably are heavily represented by sampling from the dorsally placed cannula. Thus, 3-h incubation treatments had little effect on motility of isotrichids and did not interact with capillary chemosensory compounds even when chemotaxis was blunted to the magnitude of the high $\mathrm{NO}_{2}{ }^{-}$treatment.

When comparing the main effects of chemoattractants (i.e., averaged over incubation treatments), both glucose and peptides increased $(P<0.05)$ isotrichid counts in capillary tubes, but glucose was greater $(P<$ $0.05)$ than peptides. Isotrichids are highly chemotactic toward their main substrates, glucose or other sugars, which are rapidly converted to glycogen (Diaz et al., 2014a). In contrast, peptides were chemoattractive in the current study and 1 of the 3 studies summarized by Diaz et al. (2014a) but were chemorepellent in the other 2 of those 3 previously reported studies. Although this phenomenon is not yet resolved, those authors explained that nonrumen ciliates have inconsistent attraction or repellence toward a peptide gradient as a food cue or an indication of excessive lysis. Those authors also discussed the likelihood that isotrichids probably are much less predatory toward bacteria (high in protein) than entodiniomorphids, particularly the small entodinia.

For entodiniomorphids (Figure 3, panel B), incubation beaker treatment $\times$ capillary tube treatment was $P<0.01$. Therefore, incubation beaker treatments were statistically evaluated within capillary tube treatment, and capillary tube treatments were evaluated within beaker treatments except that glucose and peptides are shown as stacked (i.e., the top of the combined bar) compared with the saline control (in common but repeated for both glucose and peptide capillary treatments in the lower gray portion of the stacked bars). The lack of incubation treatment effect $(P>0.15)$ on chemoattraction to glucose (left 6 bars in Figure 3B) is consistent with the theory by Diaz et al. (2014a) that modest, but constant, chemoattraction to glucose explains why entodiniomorphids remain attracted to, and pass with, the potentially degradable starch and fiber without specific permanent attachment to those passing particles. Hook et al. (2017) modeled protozoal passage either with the particulate phase or associated with the rumen epithelium and parameterized a loss of cell counts in the omasum. The association with epithelium is pronounced for isotrichids when they are not chemotactic, as discussed previously. Moreover, a decrease in counts could result in dilution with bypassed water or cellular lysis that does not necessarily result in lost biomass (Firkins and Yu, 2006). Protozoal biomass (especially entodiniomorphids because of their larger numbers and lack of sequestration compared with isotrichids) contributes significantly to microbial AA (especially lysine) supply to the dairy cow (Sok et al., 2017; Ahvenjärvi et al., 2018).

Entodiniomorphid chemotaxis toward peptides (right 6 bars of Figure 3B) was affected $(P \leq 0.07)$ by all contrasts except the $\mathrm{N}$ source $\times$ dosage interaction $(P$ $>0.15)$ for incubation pre-incubation treatments. However, assuming $\mathrm{NO}_{3}{ }^{-}$was being reduced to $\mathrm{NO}_{2}{ }^{-}$(probably by both bacterial and protozoal nitrate reductases) and that $\mathrm{NO}_{2}{ }^{-}$would accumulate during pre-incubation as in the companion paper (Welty et al., 2019), the overall response probably reflects an increasing doseresponse of $\mathrm{NO}_{2}^{-}$. Diaz et al. (2014a) reported that Snp stimulated chemotaxis (presumably mediated via NO signaling) by entodiniomorphids toward glucose but not toward peptides. Thus, suppression $(P \leq 0.05)$ of chemoattraction to peptides (Figure 3B) by both doses of $\mathrm{NO}_{2}{ }^{-}$and Snp is likely a result of a general blunted motility (Figure 2B). Little information is available about a potential role for $\mathrm{NO}_{2}{ }^{-}$to mimic NO-activation of protein kinase $\mathrm{G}$ in rumen or gut free-living ciliates. Because isotrichids are more aerotolerant than entodiniomorphids (Williams and Coleman, 1997), $\mathrm{NO}_{2}{ }^{-}$ or NO could be eliciting an oxidative stress response that is more acute in entodiniomorphids. Despite the slower overall motility by $\mathrm{NO}_{2}^{-}$(Figure 2B), the lack of inhibition for chemoattraction to glucose (Figure 3B, left side) compared with inhibition of chemoattraction toward peptides implies a NO-dependent chemoattrac- 


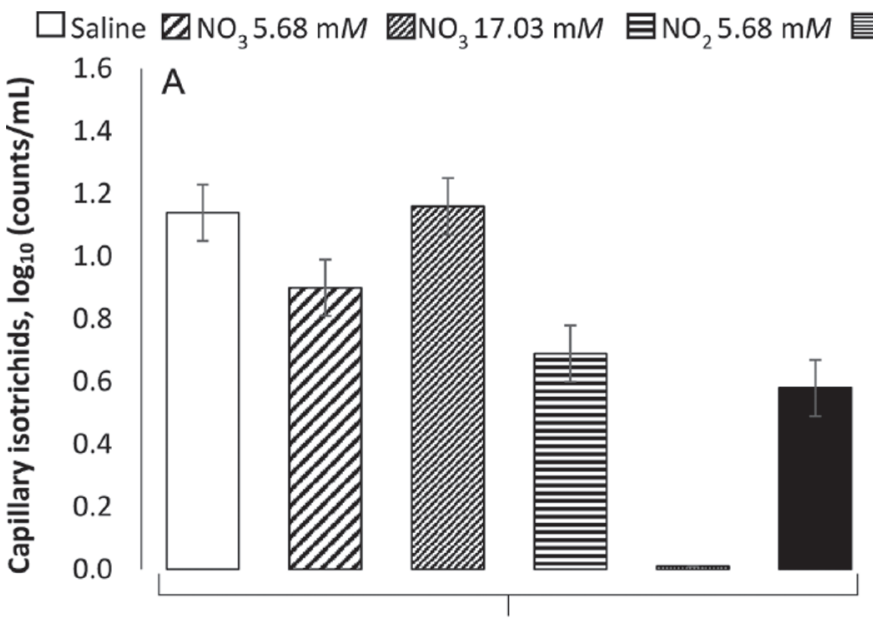

Main effect of beaker treatment
Saline 9 Glucose $\mathrm{\theta}$ Peptides

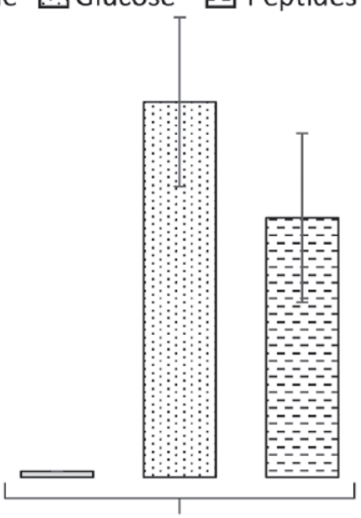

Main effect of capillary chemoattractant

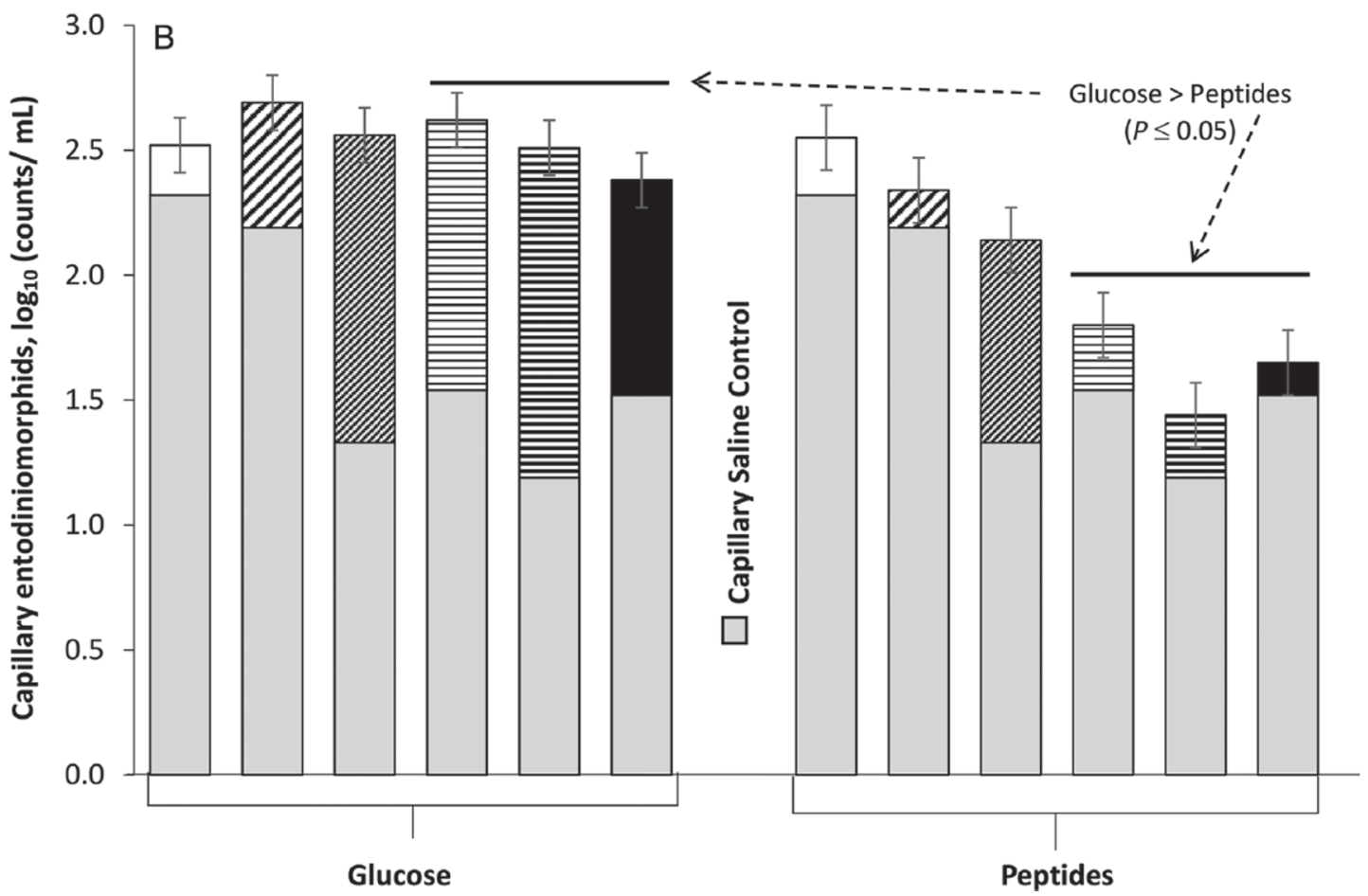

Figure 3. In experiment 2, main effect means of log-transformed counts of protozoa in capillary tubes filled with saline or saline containing $1 \mathrm{~mol} / \mathrm{L}$ glucose or $1 \mathrm{~g} / \mathrm{L}$ soluble peptides as chemoattractants for $20 \mathrm{~min}$ after capillary tubes were placed into beakers that were pre-incubated for $3 \mathrm{~h}$ with 6 treatments: (1) saline, (2) $5.68 \mathrm{~m} M \mathrm{NaNO}_{3}$, (3) $17.03 \mathrm{~m} M \mathrm{NaNO}_{3}$, (4) $5.68 \mathrm{~m} M \mathrm{NaNO}_{2}$, (5) $17.03 \mathrm{~m} M \mathrm{NaNO}_{2}$, and (6) $500 \mu M$ sodium nitroprusside $(\mathrm{Snp})$. In panel A (isotrichids), there was no interaction $(P=0.37)$ between incubation beaker treatment and capillary tube chemoattractant, so main effect means for incubation beaker treatments (left) and capillary tube treatments (right) are presented. For beaker treatment contrasts: saline versus $\left(\mathrm{NO}_{3}^{-}+\mathrm{NO}_{2}^{-}\right), 1$ versus average of 2 to $5(P<0.01)$; main effect of $\mathrm{NO}_{3}^{-}$versus $\mathrm{NO}_{2}^{-}, 2+3$ versus $4+5(P<0.01)$; main effects of dosage of $\mathrm{NO}_{3}{ }^{-}$or $\mathrm{NO}_{2}{ }^{-}, 2+4$ versus $3+5(P=0.03)$; interaction of $\mathrm{NO}_{3}{ }^{-}$versus $\mathrm{NO}_{2}{ }^{-}$and their dosages, $2+5$ versus $3+4(P<0.01)$; and saline versus Snp, 1 versus $6(P=0.04)$. For capillary tube treatments: glucose $>$ peptides $>$ saline $($ all $P<0.05)$. Error bars depict the respective pooled SEM. In panel B (entodiniomorphids), the interaction between incubation beaker treatment and capillary tube treatment was $P<0.01$. The saline capillary tube controls are represented by lower gray bars (repeated for glucose and peptide series). For saline capillary tubes, the beaker treatments: control versus average of $\mathrm{NO}_{3}^{-}+\mathrm{NO}_{2}^{-}(1$ vs. $2-5)$ was $P<0.01$; control versus Snp $(1$ vs. 6$)$ was $P<0.01$; the main effect of $\mathrm{NO}_{3}{ }^{-}$versus $\mathrm{NO}_{2}^{-}(2+3$ vs. $4+5)$ was $P<0.01$; the main effect of dosage of $\mathrm{NO}_{3}{ }^{-}$or $\mathrm{NO}_{2}{ }^{-}(2+4$ vs. $3+5)$ was $P<0.01$; the interaction $(2+5$ vs. $3+4)$ was $P>0.15$. For glucose (left 6 columns stacked above saline controls; i.e., ranging from 2.3 to 2.7 $\log$ counts), no beaker treatment had any effect $(P>0.15)$. For peptides $\left(6\right.$ stacked bars on the right): saline versus average of $\mathrm{NO}_{3}{ }^{-}+\mathrm{NO}_{2}{ }^{-}$, 1 versus 2 to $5(P<0.01)$; the main effect of $\mathrm{NO}_{3}{ }^{-}$versus $\mathrm{NO}_{2}{ }^{-}, 2+3$ versus $4+5(P=0.07)$; the main effects of dosage of $\mathrm{NO}_{3}{ }^{-}$or $\mathrm{NO}_{2}{ }^{-}, 2+4$ versus $3+5(P=0.06)$; the interaction, $2+5$ versus $3+4(P>0.15)$; and control versus Snp, 1 versus $6(P<0.01)$. Log counts in glucose versus peptides (stacked bars) were not different $(P>0.15)$ for saline control or either $\mathrm{NO}_{3}{ }^{-}$treatment; within both $\mathrm{NO}_{2}{ }^{-}$treatments and Snp, glucose $>$ peptides $(P \leq 0.05)$. 
tion toward a glucose gradient in capillary tubes. Such results would be consistent with other studies and the recognized role of cilia near the oral region in entodiniomorphids to help direct starch granules for ingestion (Jouany and Ushida, 1999; Diaz et al., 2014c).

Yang et al. (2016) reviewed the relatively few studies in which protozoa-enriched fractions (which included bacteria and archaea) increased nitrate reduction compared with bacterial (and archaeal) fractions that had protozoa removed. The isotrichid protozoa might have modest respiratory activity, although the nature of a potential terminal oxidase (cytochromes have not been detected) is not known (Williams and Coleman, 1997). Whether $\mathrm{NO}_{3}{ }^{-}$can serve as an electron sink for rumen protozoa also is not known, although the draft genome of Entodinium caudatum has a gene annotated as nitrate reductase (T. Park, The Ohio State University, Columbus, personal communication). Greater $\mathrm{NO}_{2}{ }^{-}$reduction has been detected in some protozoa-rich fractions than in bacterial fractions (Yang et al., 2016), but it is unknown if interrupted motility influences the accuracy or variability of such activity measurements.

\section{CONCLUSIONS}

Growth of a $S$. cerevisiae culture was inhibited dose-responsively by the $\mathrm{NO}_{2}{ }^{-}$concentrations used, but continued growth suggests lack of acute toxicity. Further studies are needed to ascertain if the limited growth influences the role of live yeast culture in diets supplemented with $\mathrm{NO}_{3}{ }^{-}$. Motility and chemotaxis was affected differently by $\mathrm{NO}_{2}{ }^{-}$but not $\mathrm{NO}_{3}{ }^{-}$for isotrichids versus entodiniomorphids, potentially explaining variable toxicity to $\mathrm{NO}_{2}^{-}$reported among studies. Because of the role of NO signaling in ruminal eukaryotes (including fungi, which were not studied herein), a better understanding of their $\mathrm{NO}_{2}{ }^{-}$metabolism is needed to improve the feasibility of dietary nitrates to abate methanogenesis in ruminants.

\section{ACKNOWLEDGMENTS}

Research funds were provided by Alltech Inc. (Nicholasville, KY). Research was jointly supported by state and federal funds appropriated to the Ohio Agricultural Research and Development Center, The Ohio State University. Manuscript number 39/18AS.

\section{REFERENCES}

Ahvenjärvi, S., M. Vaga, A. Vanhatalo, and P. Huhtanen. 2018. Ruminal metabolism of grass silage soluble nitrogen fractions. J. Dairy Sci. 101:279-294.
Asanuma, N., S. Yokoyama, and T. Hino. 2015. Effects of nitrate addition to a diet on fermentation and microbial populations in the rumen of goats, with special reference to Selenomonas ruminantium having the ability to reduce nitrate and nitrite. Anim. Sci. J. 86:378-384

Castello, P. R., P. S. David, T. McClure, Z. Crook, and R. O. Poyton. 2006. Mitochondrial cytochrome oxidase produces nitric oxide under hypoxic conditions: Implications for oxygen sensing and hypoxic signaling in eukaryotes. Cell Metab. 3:277-287.

Dehority, B. A. 1993. Laboratory Manual for Classification and Morphology of Rumen Ciliate Protozoa. CRC Press, Boca Raton, FL.

Dehority, B. A. 1998. Generation times of Epidinium caudatum and Entodinium caudatum, determined in vitro by transferring at various time intervals. J. Anim. Sci. 76:1189-1196.

Diaz, H. L., K. N. Barr, K. R. Godden, J. E. Plank, I. Zapata, A. N. Schappacher, M. P. Wick, and J. L. Firkins. 2014a. Eukaryotic inhibitors or activators elicit responses to chemosensory compounds by ruminal isotrichid and entodiniomorphid protozoa. J. Dairy Sci. 97:2254-2269.

Diaz, H. L., S. K. R. Karnati, M. A. Lyons, B. A. Dehority, and J. L. Firkins. 2014b. Chemotaxis toward carbohydrates and peptides by mixed ruminal protozoa when fed, fasted, or incubated with polyunsaturated fatty acids. J. Dairy Sci. 97:2231-2243.

Diaz, H. L., J. R. Knapp, S. K. R. Karnati, B. A. Dehority, and J. L. Firkins. 2014c. Effects of wortmannin, sodium nitroprusside, insulin, genistein, and guanosine triphosphate on chemotaxis and cell growth of Entodinium caudatum, Epidinium caudatum, and mixed ruminal protozoa. J. Dairy Sci. 97:2244-2253.

Firkins, J. L., and Z. Yu. 2006. Characterisation and quantification of the microbial populations in the rumen. Pages 19-54 in Ruminant Physiology, Digestion, Metabolism and Impact of Nutrition On Gene Expression, Immunology and Stress. K. Sejrsen, T. Hvelplund, and M. O. Nielsen, ed. Wageningen Academic Publishers, Wageningen, the Netherlands.

Green, S. R., and C. M. Mochle. 1999. Media and culture of yeast. Pages 1.6.1-1.6.12 in Current Protocols in Cell Biology. John Wiley \& Sons, Hoboken, NJ. https://doi.org/10.1002/0471143030 .cb0106s04.

Hinze, H., and H. Holzer. 1986. Analysis of the energy metabolism after incubation of Saccharomyces cerevisiae with sulfite or nitrite. Arch. Microbiol. 145:27-31.

Hook, S. E., J. France, and J. Dijkstra. 2017. Further assessment of the protozoal contribution to the nutrition of the ruminant animal. J. Theor. Biol. 416:8-15.

Hristov, A. N., J. Oh, J. L. Firkins, J. Dijkstra, E. Kebreab, G. Waghorn, H. P. Makkar, A. T. Adesogan, W. Yang, C. Lee, P. J. Gerber, B. Henderson, and J. M. Tricarico. 2013a. Special topicsmitigation of methane and nitrous oxide emissions from animal operations: I. A review of enteric methane mitigation options. J. Anim. Sci. 91:5045-5069.

Hristov, A. N., J. Oh, C. Lee, R. Meinen, F. Montes, T. Ott, J. Firkins, A. Rotz, C. Dell, A. Adesogan, W. Yang, J. Tricarico, E. Kebreab, G. Waghorn, J. Dijkstra, and S. Oosting. 2013b. Mitigation of greenhouse gas emissions in livestock production-A review of technical options for non- $\mathrm{CO}_{2}$ emissions. Animal Production and Health Paper No. 177. FAO, Rome, Italy 190:95-96.

Jeyanathan, J., C. Martin, and D. P. Morgavi. 2014. The use of directfed microbials for mitigation of ruminant methane emissions: A review. Animal 8:250-261.

Jouany, J. P., and K. Ushida. 1999. The role of protozoa in feed digestion-Review. Asian-australas. J. Anim. Sci. 12:113-128.

Latham, E. A., R. C. Anderson, W. E. Pinchak, and D. J. Nisbet. 2016. Insights on alterations to the rumen ecosystem by nitrate and nitrocompounds. Front. Microbiol. 7:228.

Ling, W. C., Y. S. Lau, D. D. Murugan, P. M. Vanhoutte, and M. R. Mustafa. 2015. Sodium nitrite causes relaxation of the isolated rat aorta: By stimulating both endothelial no synthase and activating soluble guanylyl cyclase in vascular smooth muscle. Vascul. Pharmacol. 74:87-92.

Lund, P., R. Dahl, H. J. Yang, A. L. F. Hellwing, B. B. Cao, and M. R. Weisbjerg. 2014. The acute effect of addition of nitrate on in 
vitro and in vivo methane emission in dairy cows. Anim. Prod. Sci. 54:1432-1435.

Newbold, C. J., R. J. Wallace, and F. M. McIntosh. 1996. Mode of action of the yeast Saccharomyces cerevisiae as a feed additive for ruminants. Br. J. Nutr. 76:249-261.

Park, T., T. Meulia, J. L. Firkins, and Z. Yu. 2017. Inhibition of the rumen ciliate Entodinium caudatum by antibiotics. Front. Microbiol. 8:1189.

Sarver, A., and J. DeRisi. 2005. Fzf1p regulates an inducible response to nitrosative stress in Saccharomyces cerevisiae. Mol. Biol. Cell 16:4781-4791.

Schneider, C. A., W. S. Rasband, and K. W. Eliceiri. 2012. NIH image to ImageJ: 25 years of image analysis. Nat. Methods 9:671.

Sok, M., D. R. Ouellet, J. L. Firkins, D. Pellerin, and H. Lapierre. 2017. Amino acid composition of rumen bacteria and protozoa in cattle. J. Dairy Sci. 100:5241-5249.
Welty, C. M., B. A. Wenner, B. K. Wagner, Y. Roman-Garcia, J. E. Plank, R. A. Meller, A. M. Gehman, and J. L. Firkins. 2019. Rumen microbial responses to supplemental nitrate. II. Potential interactions with live yeast culture on the prokaryotic community and methanogenesis in continuous culture. J. Dairy Sci. 102:22172231. https://doi.org/10.3168/jds.2018-15826.

Wenner, B. A., B. K. Wagner, and J. L. Firkins. 2018. Using video microscopy to improve quantitative estimates of protozoal motility and cell volume. J. Dairy Sci. 101:1060-1073.

Williams, A. G., and G. S. Coleman. 1997. The rumen protozoa. Pages 73-139 in The Rumen Microbial Ecosystem. P. N. Hobson and C. S. Stewart, ed. Chapman \& Hall, London, UK.

Yang, C., J. A. Rooke, I. Cabeza, and R. J. Wallace. 2016. Nitrate and inhibition of ruminal methanogenesis: Microbial ecology, obstacles, and opportunities for lowering methane emissions from ruminant livestock. Front. Microbiol. 7:132. 